\title{
FOXA1 Induces E-Cadherin Expression at the Protein Level via Suppression of Slug in Epithelial Breast Cancer Cells
}

\author{
Erina Anzai, Kensuke Hirata, Misato Shibazaki, Chiaki Yamada, Mariko Morii, Takuya Honda, \\ Naoto Yamaguchi, and Noritaka Yamaguchi* \\ Laboratory of Molecular Cell Biology, Graduate School of Pharmaceutical Sciences, Chiba University; 1-8-1 \\ Inohana, Chuo-ku, Chiba 260-8675, Japan. \\ Received April 12, 2017; accepted June 7, 2017
}

\begin{abstract}
Epithelial-to-mesenchymal transition (EMT) is an important process during embryonic development and tumor progression by which adherent epithelial cells acquire mesenchymal properties. Forkhead box protein A1 (FOXA1) is a transcriptional regulator preferentially expressed in epithelial breast cancer cells, and its expression is lost in mesenchymal breast cancer cells. However, the implication of this biased expression of FOXA1 in breast cancer is not fully understood. In this study, we analyzed the involvement of FOXA1 in EMT progression in breast cancer, and found that stable expression of FOXA1 in the mesenchymal breast cancer MDA-MB-231 cells strongly induced the epithelial marker E-cadherin at the mRNA and protein levels. Furthermore, stable expression of FOXA1 was found to reduce the mRNA and protein expression of Slug, a repressor of E-cadherin expression. FOXA1 knockdown in the epithelial breast cancer MCF7 cells reduced E-cadherin protein expression without decreasing its mRNA expression. In addition, FOXA1 knockdown in MCF7 cells up-regulated Slug mRNA and protein expression. Notably, similar to FOXA1 knockdown, stable expression of Slug in MCF7 cells reduced E-cadherin protein expression without decreasing its mRNA expression. Taken together, these results suggest that although FOXA1 can induce E-cadherin mRNA expression, it preferentially promotes E-cadherin expression at the protein level by suppressing Slug expression in epithelial breast cancer, and that the balance of this FOXA1-Slug axis regulates EMT progression.
\end{abstract}

Key words epithelial-mesenchymal transition; E-cadherin; forkhead box protein A1; Slug; estrogen receptor

Epithelial-to-mesenchymal transition (EMT) is a process that converts adherent epithelial cells to motile mesenchymal cells during embryonic development. ${ }^{1)}$ EMT is also involved in tumor progression through inducing metastasis and chemoresistance in tumor cells. ${ }^{2-4)}$ Therefore, a better understanding of the molecular mechanisms underlying EMT in tumor cells is beneficial for development of novel antitumor agents.

E-Cadherin is a crucial protein that mediates cell-cell adhesion in epithelial cells. ${ }^{1)}$ E-Cadherin expression is dramatically reduced during EMT, which causes loss of cell-cell adhesion and gain of cell motility. Reduction in E-cadherin expression is mainly caused by the transcriptional repressors of E-cadherin gene $(C D H 1){ }^{2,5)}$ A major transcriptional repressor of $C D H 1$ is the zinc finger factor Slug. Slug binds to the E-box sequences present in the enhancer region of $C D H 1$ and represses its expression. ${ }^{6)}$

Forkhead box A1 (FOXA1) is a member of the forkhead box family of transcription factors. FOXA1 contains the forkhead DNA-binding domain and the N- and C-terminal transactivation domains. ${ }^{7)}$ FOXA1 regulates expression of many genes involved in the development of various tissues, including mammary gland, liver, midbrain, and lung. ${ }^{8)}$ FOXA1 is also known to function as a chromatin remodeling factor that opens closed chromatin and facilitates the recruitment of other transcription factors. ${ }^{9)}$ Tyrosine phosphorylation in the C-terminal region of FOXA1 was shown to enhance this chromatin remodeling activity. ${ }^{10)}$ Because estrogen receptor- $\alpha$ $(\mathrm{ER} \alpha)$ depends on this chromatin remodeling activity, FOXA1 is crucial for the development of not only normal mammary gland but also estrogen-dependent breast cancer. ${ }^{11,12)}$

Recent studies have shown that FOXA1 suppresses EMT progression in pancreatic and prostate cancer. ${ }^{13,14)}$ In breast cancer, FOXA1 is preferentially expressed in estrogen-dependent ER $\alpha$-positive epithelial breast cancer cells, whereas its expression is lost in estrogen-independent ER $\alpha$-negative mesenchymal breast cancer cells. ${ }^{15)}$ FOXA1 expression is associated with good prognosis in breast cancer. ${ }^{16)}$ These results suggest that although FOXA1 is crucial for proliferation of estrogen-dependent ER $\alpha$-positive epithelial breast cancer cells, FOXA1 suppresses conversion from less malignant epithelial cancer to more aggressive mesenchymal cancer. However, the involvement of FOXA1 in EMT progression in breast cancer is not fully understood. Therefore, we designed this study to analyze the role of FOXA1 in EMT in breast cancer cells.

\section{MATERIALS AND METHODS}

Plasmids Plasmids encoding FOXA1 and ER $\alpha$ were generated as described previously. ${ }^{17)}$ cDNA encoding Slug was generated by PCR from the reverse-transcribed product of MDA-MB-231 total RNA and was subcloned into the pIRESpuro3 vector (TaKaRa, Shiga, Japan). A myc tag was added to the N-terminal cDNA of Slug.

Antibodies The following antibodies were used: FOXA1 (sc-6553) and p53 (sc-126) from Santa Cruz Biotechnology (Santa Cruz, CA, U.S.A.); ER $\alpha$ (\#8644), E-cadherin (\#3195), Slug (\#9585), and vimentin (\#5741) from Cell Signaling Technology (Beverly, MA, U.S.A.); LC3 (PM036) from MBL (Nagoya, Japan); and actin (MAB1501) from Merck Millipore (Guyancourt, France). Horseradish peroxidase (HRP)-F( $\left(\mathrm{ab}^{\prime}\right)_{2}$ secondary antibodies were purchased from GE Healthcare (Waukesha, WI, U.S.A.). 
Cells and Transfection MCF7 and MDA-MB-231 cells were cultured in Dulbecco's modified Eagle's medium (Dulbecco's modified Eagle's medium (DMEM); Nissui, Tokyo, Japan) containing 5\% fetal bovine serum. MG-132 and bafilomycin A1 were purchased from Peptide Institute (Osaka, Japan) and Cayman Chemical (Ann Arbor, MI, U.S.A.), respectively. For plasmid transfection, cells were seeded in a 35-mm (or 60-mm) culture dish and transiently transfected with $1 \mu \mathrm{g}$ (or $3 \mu \mathrm{g}$ ) of plasmid DNA using Lipofectamine 2000 (Life Technologies, Carlsbad, CA, U.S.A.) according to the manufacturer's instructions. To establish stable cell pools, cells were transfected with pIRESpuro3 vectors and selected with puromycin $(1 \mu \mathrm{g} / \mathrm{mL})$ for $2 \mathrm{wk}$. Puromycin-resistant cells were used as stable cell pools. Small interfering RNA (siRNA) for FOXA1 \#1 (sense: 5'-GAA CAGGCA CUGCAA UAC UCG CCUU-3' and anti-sense: 5'-AAGGCGAGU AUU GCA GUG CCU GUU C-3') was purchased from Life Technologies. siRNAs for FOXA1 \#2 (sense: 5'-GAGAGA AAAAAUCAA CAGC-3' and anti-sense: 5'-GCUGUUGAUUUUUUCUCUC-3') and luciferase (sense: 5'-GCGCUG CUGGUGCCA ACC CTT-3' and anti-sense: 5'-GGGUUG GCA CCA GCA GCGCTT-3') were purchased from Hokkaido System Sciences (Hokkaido, Japan). siRNAs were transfected into cells using Lipofectamine 2000 according to the manufacturer's instructions.

Western Blotting Western blotting was performed by enhanced chemiluminescence (Merck Millipore) as described previously. ${ }^{17)}$ Whole cell lysates prepared in sodium dodecyl sulfate (SDS) sample buffer were subjected to SDS-polyacrylamide gel electrophoresis and electrotransferred onto polyvinylidene difluoride membranes. Protein bands were detected with appropriate antibodies and analyzed using a ChemiDoc XRS+ image analyzer (Bio-Rad, Richmond, CA, U.S.A.). Intensity of chemiluminescence was measured using Quantity One software (Bio-Rad).

Quantitative Real-Time PCR (qPCR) Analysis qPCR was performed as described previously. ${ }^{18)}$ Briefly, total RNA was isolated from cells using RNAiso Plus (TaKaRa), and cDNA was synthesized from $0.5 \mu \mathrm{g}$ of each RNA preparation using a ReverTra Ace qPCR RT Kit (TOYOBO, Tokyo, Japan), according to the manufacturer's instructions. The primers used for PCR were as follows: glyceraldehyde 3-phosphate dehydrogenase $(G A P D H)$, 5'-ACC ACA GTCCAT GCC ATC AC-3' (sense) and 5'-TCC ACC ACC CTG TTGCTGTA-3' (antisense); E-cadherin (CDH1), 5'-CGA GAGCTACAC GTT CACGG-3' (sense) and 5'-GGG TGT CGA GGGAAA AAT AGG-3' (antisense); Snail (SNAI1), 5'-TCGGAA GCC TAA CTACAGCGA-3' (sense) and 5'-AGA TGA GCA TTGGCA GCG AG-3' (antisense); Slug (SNAI2), 5'-TGT GAC AAGGAA TAT GTGAGCC-3' (sense) and 5'-TGA GCCCTCAGA TTT GACCTG-3' (antisense); FOXA1 (FOXA1), 5'-GCAATACTC GCC TTA CGGCT-3' (sense) and 5'-TAC ACA CCT TGG TAG TACGCC-3' (antisense). After initial denaturation at $95^{\circ} \mathrm{C}$ for $1 \mathrm{~min}, \mathrm{PCR}$ was performed for 40 cycles $\left(15 \mathrm{~s}\right.$ at $95^{\circ} \mathrm{C}$ and $45 \mathrm{~s}$ at $60^{\circ} \mathrm{C}$ ) by using a Thunderbird SYBR Green Polymerase Kit (TOYOBO) and an Eco Real-Time PCR System (Illumina, San Diego, CA, U.S.A.).

Immunofluorescence Confocal and Nomarski differential-interference-contrast (DIC) images were obtained with a Fluoview FV500 confocal laser scanning microscope (Olympus, Tokyo, Japan), as described. ${ }^{19)}$ In brief, cells were fixed in $4 \%$ paraformaldehyde for $20 \mathrm{~min}$ at room temperature and permeabilized in phosphate-buffered saline (PBS) containing $0.2 \%$ Triton $\mathrm{X}-100$ and $3 \%$ bovine serum albumin. Cells were subsequently reacted with anti-Slug antibody (\#9585) for $1 \mathrm{~h}$, washed with PBS containing $0.1 \%$ saponin, stained with Alexa Fluor 488-conjugated secondary antibody for $1 \mathrm{~h}$, and mounted with ProLong antifade reagent (Life Technologies). Nuclei were stained with TO-PRO-3 (Life Technologies).

Wound Healing Assay Cells were seeded in a $35-\mathrm{mm}$ culture dish and cultured until they reached $100 \%$ confluency. Wounds of equal width were generated in confluent cell layers using a pipette tip. Images of the wound healing process were captured with a phase-contrast microscope after the indicated periods. Invaded area, measured using the ImageJ software, was calculated as follows: percentage of invaded area $=[1-($ wounded area at the indicated time $) /($ wounded area at $0 \mathrm{~h})] \times 100$.

\section{RESULTS}

FOXA1 Induces E-Cadherin Expression Independently of ER $\alpha$ In this study, we used MCF7 cells as a model of estrogen-dependent ER $\alpha$-positive epithelial breast cancer and MDA-MB-231 cells as a model of estrogen-independent $\mathrm{ER} \alpha$-negative mesenchymal breast cancer. Expression of ER $\alpha$, its transcriptional partner FOXA1, and the epithelial marker E-cadherin was detected in MCF7 cells, but not in MDAMB-231 cells (Supplementary Fig. S1A). In contrast, the mesenchymal marker Slug was expressed only in MDA-MB-231 cells. MCF7 cells showed cell-cell adhesion with cobblestonelike morphology, whereas MDA-MB-231 cells lacked cell-cell adhesion with spindle-like morphology (Supplementary Fig. S1B). Wound healing assays showed that MDA-MB-231 cells had higher motility than MCF7 cells (Supplementary Fig. S1C). These results showed that MCF7 cells exhibited characteristics of estrogen-dependent ER $\alpha$-positive epithelial breast cancer, whereas MDA-MB-231 cells had those of estrogenindependent $\mathrm{ER} \alpha$-negative mesenchymal breast cancer.

Because previous studies have shown that ER $\alpha$ and FOXA1 are involved in E-cadherin expression, ${ }^{20,21)}$ we investigated the genes important for E-cadherin expression in breast cancer cells by stable expression of each gene in MDA-MB-231 cells. Although E-cadherin expression was profoundly induced at the mRNA and protein levels by stable expression of FOXA1, ER $\alpha$ expression did not affect E-cadherin expression (Figs. 1A, B). We also found that Slug expression was significantly reduced at the mRNA and protein levels by stable expression of FOXA1 (Figs. 1A, B). Expression of the Slug homolog Snail was not affected by stable expression of each gene. Wound healing assays showed that FOXA1 expression significantly reduced the motility of MDA-MB-231 cells (Fig. 1C). FOXA1 expression changed spindle-like morphology into shrinked morphology in MDA-MB-231 cells (Fig. 1D). These results suggest that FOXA1, but not $\mathrm{ER} \alpha$, plays an important role in suppression of EMT in breast cancer cells via induction of E-cadherin expression and inhibition of Slug expression.

FOXA1 Suppresses Slug Expression in Breast Cancer Cells We next analyzed the role of endogenous FOXA1 in suppression of EMT. siRNA-mediated depletion of endogenous FOXA1 caused a significant reduction in E-cadherin expression at the protein level in MCF7 cells (Figs. 2A, B). However, E-cadherin mRNA expression was slightly increased, rather 
A

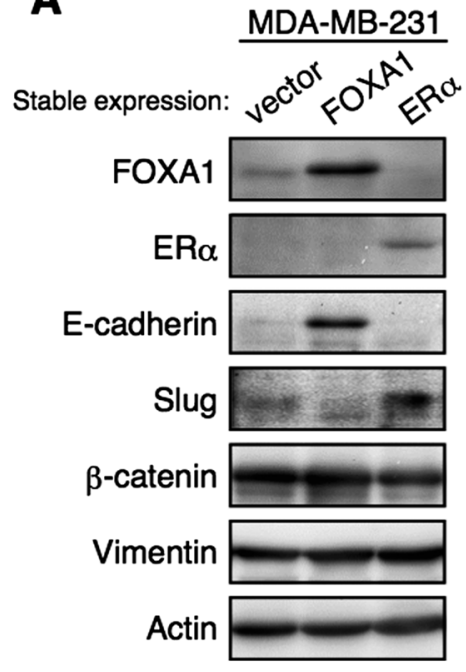

B

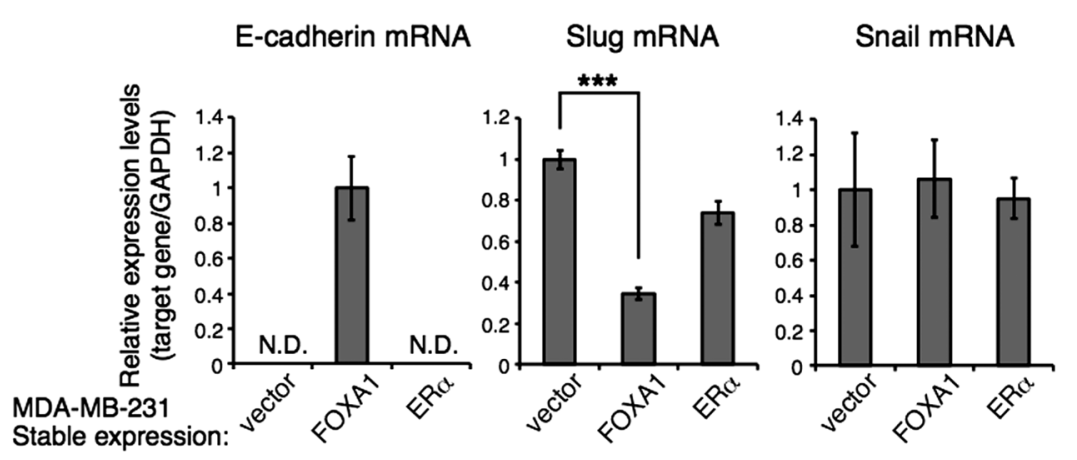

C

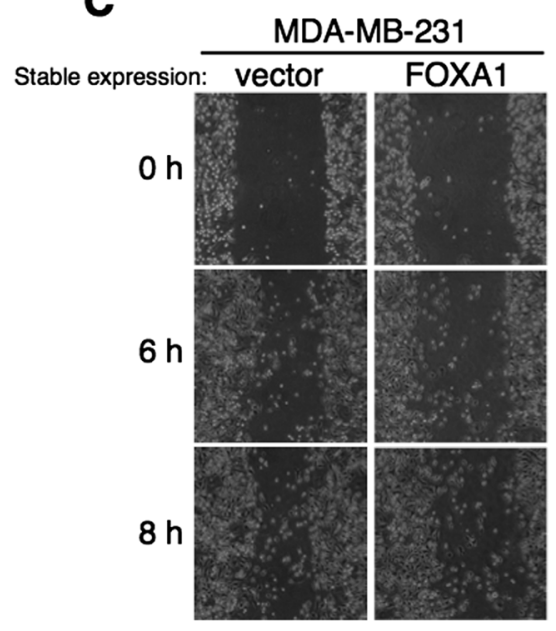

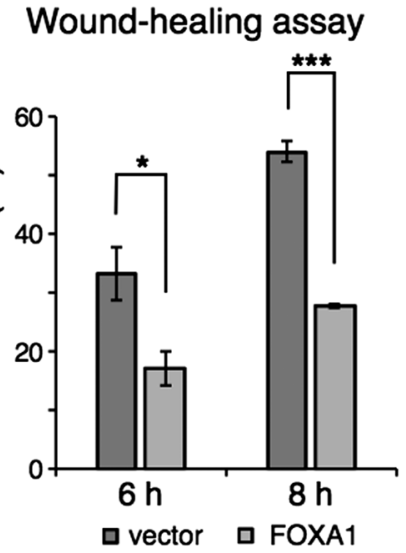

D

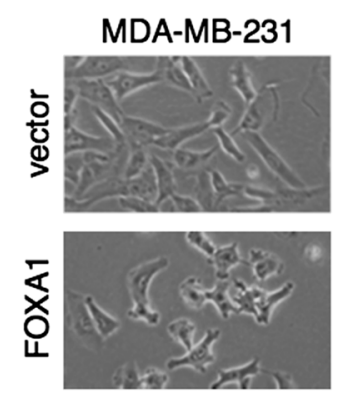

Fig. 1. Stable Expression of FOXA1 Induced E-Cadherin Expression in MDA-MB-231 Cells

(A) MDA-MB-231 cells stably expressing FOXA1, ER $\alpha$, or empty vector (pIRESpuro3) were lysed and subjected to Western blotting with the indicated antibodies. (B) MDA-MB-231 cells stably expressing FOXA1, ER $\alpha$, or empty vector were analyzed for E-cadherin, Slug, or Snail mRNA expression by qPCR analysis. GAPDH mRNA expression level was used to normalize the data. The expression level in the control cells (Slug and Snail mRNA) or in the FOXA1-expressing cells (E-cadherin mRNA) was set to 1 . Results are the mean \pm S.D. $(n=3)$. Asterisks indicate significant differences $(* * * p<0.001)$ determined by the Student's $t$-test. N.D.: Not detected. (C) Wound healing assays of MDA-MB-231 cells stably expressing FOXA1 or the empty vector. Representative images of wound healing after incubation for 0 , 6 , or $8 \mathrm{~h}$ (left). Percentage of invaded area was measured using the ImageJ software, and results are shown as the mean \pm S.D. $(n=3)$. Asterisks indicate significant differences $(* * * p<0.001$, $\left.*_{p}<0.05\right)$ determined by the Student's $t$-test (right). (D) MDA-MB-231 cells stably expressing FOXA1 or empty vector were visualized by phase-contrast microscopy.

than decreased, by FOXA1 depletion (Fig. 2C). These results suggest that although FOXA1 induces E-cadherin expression in epithelial breast cancer MCF7 cells, FOXA1 does not function as a transcriptional inducer for $C D H 1$ in these cells.

Because FOXA1 has been reported to suppress Slug expression, ${ }^{14)}$ we analyzed the involvement of FOXA1 in Slug expression in MCF7 cells. Knockdown of FOXA1 was found to strongly induce Slug expression at the mRNA and protein levels (Figs. 2A-C). Although Snail mRNA expression was increased by FOXA1 knockdown, this increase was very weak (Fig. 2C). These results suggest that FOXA1 mainly functions as a transcriptional repressor for Slug gene (SNAI2) in MCF7 cells. To investigate the effects of Slug on E-cadherin expression in MCF7 cells, we stably expressed Slug in these cells (Fig. 3A). Notably, similar to FOXA1-depleted MCF7 cells, Slug expression significantly suppressed E-cadherin protein level without decreasing its mRNA level in Slug-expressing cells (Figs. 3B-D). These results suggest that the reduction in
E-cadherin expression is mediated by Slug in FOXA1-depleted MCF7 cells and that Slug suppresses E-cadherin expression independently of its transcriptional repression activity.

Slug Represses E-Cadherin Expression at the Post-transcriptional Level in Breast Cancer Cells Slug is known to repress E-cadherin mRNA expression and promote EMT progression. ${ }^{6}$ However, Slug expression in MCF7 cells reduced E-cadherin protein level without decreasing its mRNA level. Therefore, we hypothesized that Slug represses E-cadherin protein translation or promotes protein degradation (Fig. 4A). To test the possibility that Slug promotes E-cadherin degradation, we treated Slug-expressing MCF7 cells with the proteasomal inhibitor MG-132 or the lysosomal inhibitor bafilomycin $\mathrm{A} 1$ and then analyzed accumulation of E-cadherin (Figs. 4B, C). However, E-cadherin accumulation induced by each inhibitor was not changed in Slug-expressing MCF7 cells compared with the control cells, suggesting that Slug did not increase proteasome- or lysosome-mediated E-cadherin degra- 
A

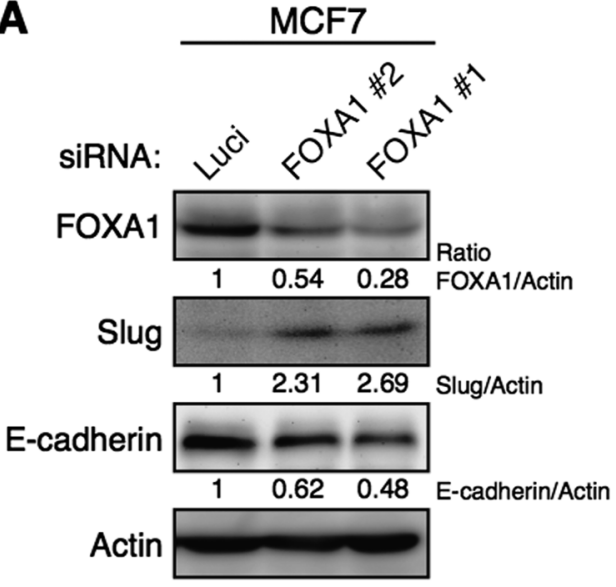

B

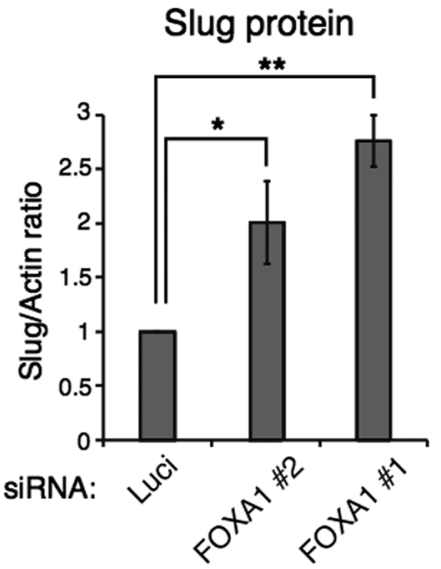

C

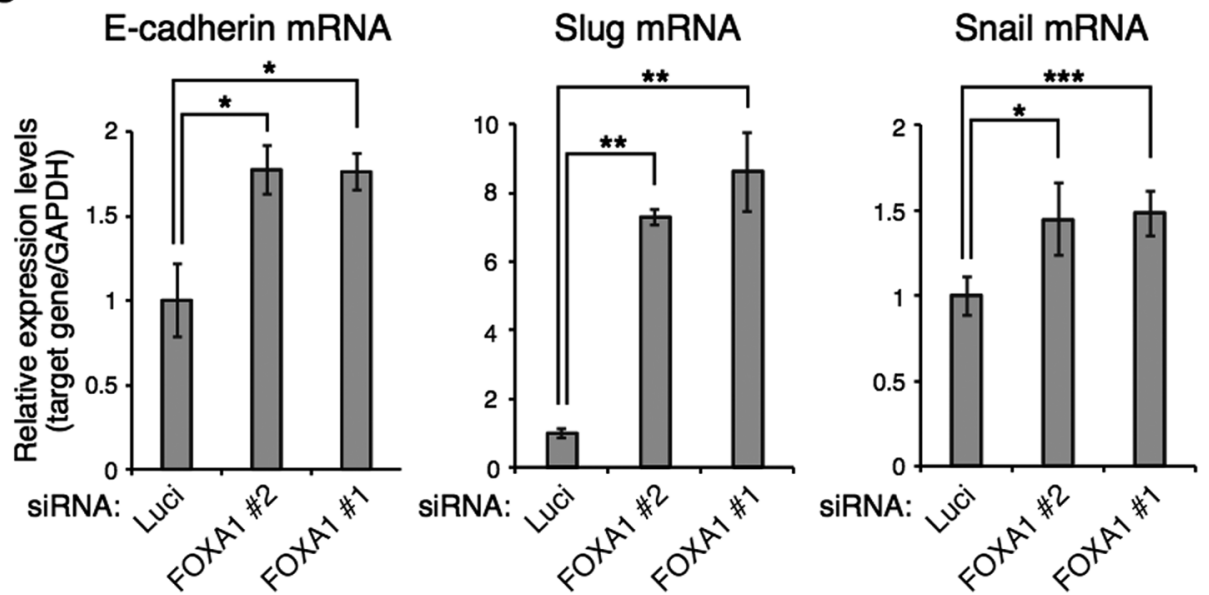

Fig. 2. FOXA1 Knockdown Reduced E-Cadherin Protein Expression without Decreasing Its mRNA Expression in MCF7 Cells

(A, B) MCF7 cells transfected with siRNAs for FOXA1 (\#1, \#2) or luciferase (Luci) were incubated for $48 \mathrm{~h}$. After incubation, cells were lysed and subjected to Western blotting with the indicated antibodies (A). Quantitative data of E-cadherin or Slug expression are shown as the mean \pm S.D. ( $n=3$ ). Asterisks indicate significant differences $(* * p<0.01, * p<0.05$ ) determined by the Student's $t$-test (B). (C) MCF7 cells transfected with siRNAs for FOXA1 (\#1, \#2) or luciferase (Luci) were incubated for $72 \mathrm{~h}$. After incubation, cells were analyzed for E-cadherin, Slug, or Snail mRNA expression by qPCR analysis as in Fig. 1B, except that the expression level in the siLucitransfected cells was set to 1 . Asterisks indicate significant differences $\left(* * * p<0.001, * * p<0.01,{ }^{*} p<0.05\right)$ determined by the Student's $t$-test.

dation. Similar to Slug-expressing cells, E-cadherin accumulation induced by MG-132 did not increase in FOXA1-depleted MCF7 cells (Fig. 4D). Taken together, these results suggest that Slug suppresses E-cadherin expression probably through repression at the translational level in epithelial breast cancer MCF7 cells.

\section{DISCUSSION}

The transcription factor FOXA1 binds to genomic DNA via the forkhead DNA-binding domain and regulates expression of various genes. ${ }^{7)}$ The promoter region of $C D H 1$ has FOXA1binding sites, and $\mathrm{CDH} 1$ expression was shown to be directly induced by FOXA1. ${ }^{20)}$ FOXA1 is also known to act as a transcriptional repressor. In epithelial breast cancer cells, FOXA1 represses expression of genes involved in malignant tumor phenotypes, and depletion of FOXA1 increases cellular motility. ${ }^{15)}$ In prostate cancer cells, FOXA1 binds to the genomic region of SNAI2 and represses its expression. ${ }^{14)}$

In this study, we showed that FOXA1 induces E-cadherin expression in breast cancer cells. Notably, FOXA1 promoted E-cadherin expression at the mRNA and protein levels in mesenchymal MDA-MB-231 cells, whereas FOXA1 induced E-cadherin expression without affecting its mRNA expression in epithelial MCF7 cells. These results suggest that there is a difference in the mechanisms underlying FOXA1-mediated expression of E-cadherin between epithelial and mesenchymal breast cancer cells. In epithelial breast cancer MCF7 cells, FOXA1-knockdown induced expression of the E-cadherin repressor Slug. Stable expression of Slug in MCF7 cells reduced E-cadherin protein expression without reducing its mRNA expression or promoting its protein degradation. Because the effects of FOXA1 depletion and Slug expression on E-cadherin expression are similar in MCF7 cells, we hypothesize that FOXA1 induces E-cadherin expression primarily through suppression of Slug in epithelial breast cancer cells (Supplementary Fig. S2).

Although Slug is known as a transcriptional repressor of $\left.\mathrm{CDH1},{ }^{6}\right)$ our present data showed that Slug reduced Ecadherin protein level without reducing its mRNA level in MCF7 cells, suggesting that Slug suppresses E-cadherin expression independently of transcriptional repression in these cells. Because proteasome- or lysosome-mediated E-cadherin degradation was not increased by Slug expression, we primar- 


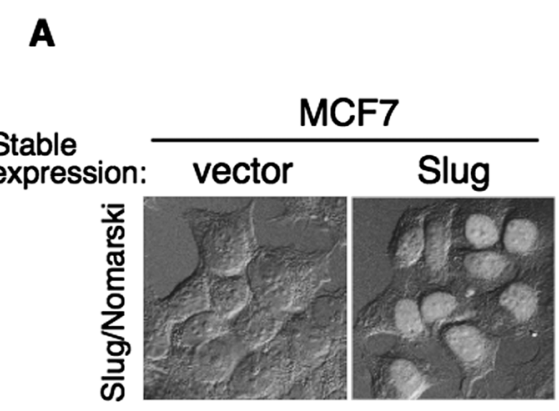

B

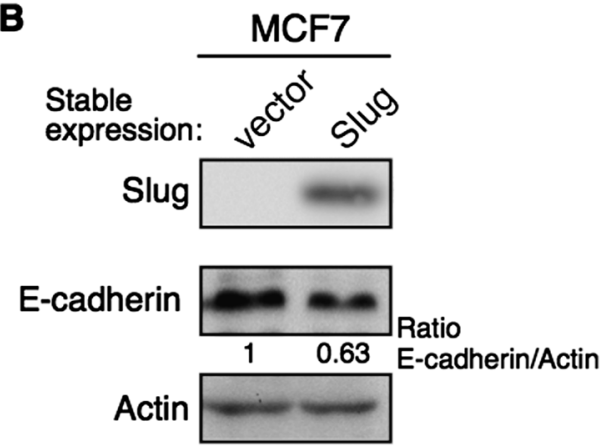

C

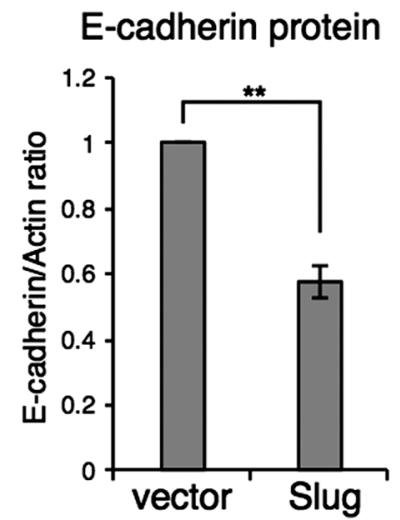

D E-cadherin mRNA

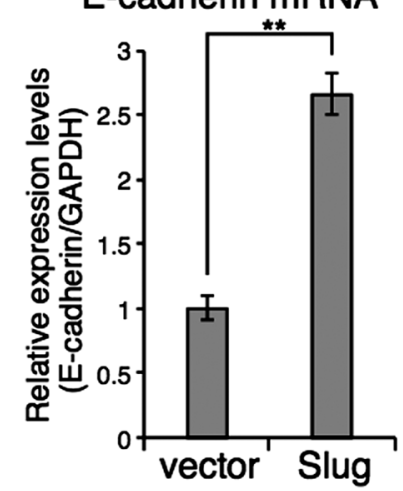

Fig. 3. Stable Expression of Slug Reduced E-Cadherin Protein Expression without Decreasing Its mRNA Expression in MCF7 Cells

(A) MCF7 cells stably expressing myc-Slug or empty vector (pIRESpuro3) were fixed and stained with anti-Slug antibody (white). Fluorescent and Nomarski DIC images were merged. (B) MCF7 cells stably expressing myc-Slug or empty vector were lysed and subjected to Western blotting with the indicated antibodies. (C) Quantitative data for E-cadherin expression are shown as the mean \pm S.D. $(n=3)$. Asterisks indicate significant differences $(* * p<0.01)$ determined by the Student's $t$-test. (D) MCF7 cells stably expressing myc-Slug or empty vector were analyzed for E-cadherin mRNA expression by qPCR analysis as in Fig. 1B. Asterisks indicate significant differences $(* * p<0.01)$ determined by the Student's $t$-test.

ily hypothesize that Slug inhibits E-cadherin expression at the translational level. Transcriptional repression is mainly caused by microRNAs, which suppress translation by binding to 3 -untranslated regions (UTRs) of target mRNAs. ${ }^{22)}$ Notably, recent studies have shown that Slug induces expression of microRNA-221 (miR-221) and suppresses E-cadherin protein translation via this microRNA. ${ }^{23)}$ Although we have not yet analyzed expression of miR-221 in FOXA1-depleted or Slug-expressing MCF7, we speculate that miR-221 is induced by Slug and then suppresses the translation of E-cadherin in these cells. However, our present data showed that E-cadherin mRNA expression was increased, rather than decreased, in FOXA1-depleted or Slug-expressing MCF7 cells, and this phenomenon was not observed in miR-221-expressing MCF7 cells. $^{23)}$ Therefore, we hypothesize that a feedback mechanism that compensates E-cadherin protein reduction drives E-cadherin mRNA expression in these MCF7 cells. We also have another hypothesis is that Slug induces proteasome- or lysosome-independent E-cadherin protein degradation. To examine the involvement of such proteasome- or lysosomeindependent degradation mechanism, E-cadherin protein halflife should be analyzed with the translational inhibitor cycloheximide in FOXA1-depleted or Slug-expressing cells.

Previous studies showed that FOXA1 forms a complex with $\operatorname{ER} \alpha$ and histone deacetylase 7 to suppress expression of reprimo gene $(R P R M)$ in breast cancer cells. ${ }^{24)}$ However, our present results showed that stable expression of FOXA1 in ER $\alpha$-negative breast cancer MDA-MB-231 cells suppressed
Slug expression, suggesting that FOXA1 regulates expression of this gene independently of estrogen receptors. Similarly, although FOXA1 is indispensable for androgen receptor-mediated gene expression, FOXA1 suppresses SNAI2 expression independently of androgen receptor in prostate cancer cells. ${ }^{14)}$ These results suggest that FOXA1 represses gene expression both dependently and independently of estrogen receptors. Recently, we found that FOXA1 suppresses binding of the transcription factor nuclear factor- $\kappa \mathrm{B}(\mathrm{NF}-\kappa \mathrm{B})$ to the promoter region of interleukin (IL)-6 gene and represses its expression independently of $\operatorname{ER} \alpha$ in breast cancer cells. ${ }^{17)}$ Therefore, we hypothesize that FOXA1 binds to the promoter region of SNAI2 and suppresses association of an undefined transcription factor for $S N A I 2$ independently of estrogen receptors.

Expression of FOXA1 in MDA-MB-231 cells suppressed Slug expression but not Snail expression (Figs. 1A, B), whereas FOXA1 knockdown increased both Slug and Snail expression in MCF7 cells (Figs. 2A-C), suggesting that Slug and Snail expression is differently regulated by FOXA1. Although the exact mechanism is unclear, we hypothesize that Snail expression is driven by multiple mechanisms, which are insensitive to FOXA1-mediated repression, in MDA-MB-231 cells to maintain strong mesenchymal phenotypes.

In conclusion, our present results showed that FOXA1 suppresses EMT progression probably through suppression of Slug expression in epithelial breast cancer cells. Because FOXA1 is required for proliferation of estrogen-dependent epithelial breast cancer cells, FOXA1 may be a therapeutic 
A

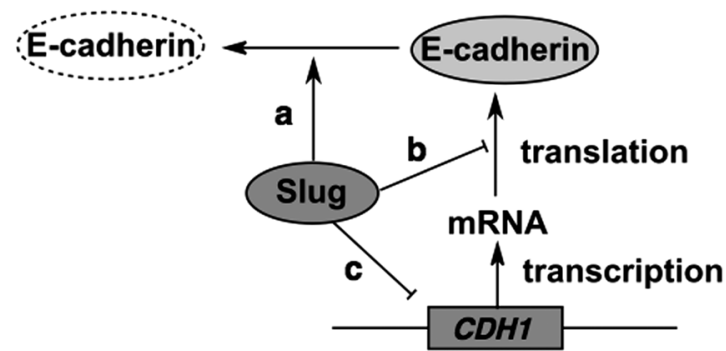

a. protein destabilization

b. translational inhibition

c. transcriptional inhibition

B

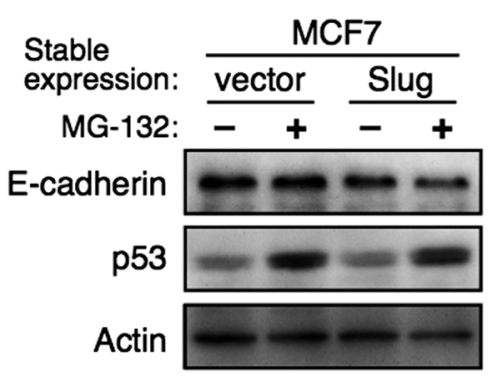

C

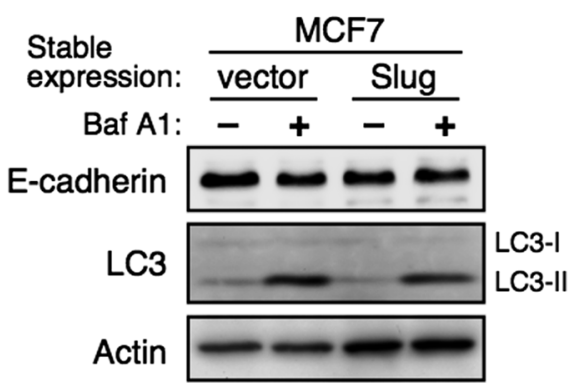

D MCF7

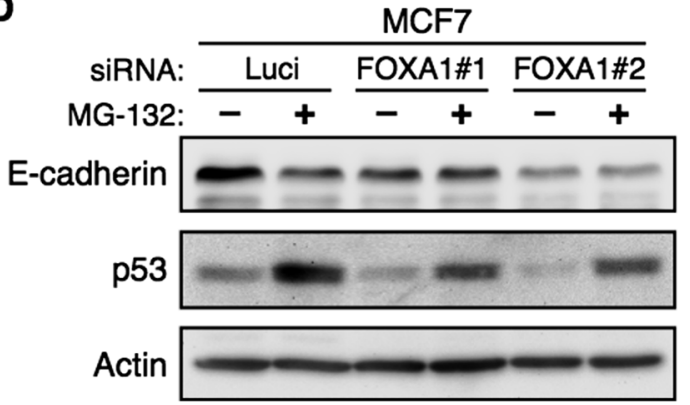

Fig. 4. Slug Expression Did Not Affect Proteasome- or Lysosome-Mediated E-Cadherin Protein Degradation in MCF7 Cells

(A) Schematic model of Slug-mediated reduction in E-cadherin expression. (B, C) MCF7 cells stably expressing myc-Slug or empty vector were left untreated or were treated with MG-132 $(20 \mu \mathrm{M}, 2 \mathrm{~h})(\mathrm{B})$ or bafilomycin A1 (Baf A1, 200nM, 2h) (C). After treatment, cells were lysed and subjected to Western blotting with the indicated antibodies. p53 and LC3-II are shown as the positive controls for MG-132- and Baf A1-treatment, respectively. (D) MCF7 cells were transfected with siRNAs for FOXA1 (\#1, \#2) or luciferase for $48 \mathrm{~h}$. After incubation, cells were left untreated or were treated with MG-132 $(20 \mu \mathrm{M}, 2 \mathrm{~h})$, lysed, and then subjected to Western blotting with the indicated antibodies.

target for this type of cancer. However, our present results suggest that suppression of FOXA1 can induce EMT progression via Slug induction in epithelial breast cancer. Therefore, dual inhibition of FOXA1 and Slug is expected to suppress both proliferation and EMT progression of epithelial breast cancer cells and may be a promising therapeutic strategy for this type of cancer.

Acknowledgments This work was supported in part by Grants-in-Aid for Scientific Research C [Grant numbers 16K08227 (to Noritaka Y.) and 15K07922 (to Naoto Y.)] and Program for Leading Graduate Schools (Leading Graduate School Chiba University Nurture of Creative Research Leaders in Immune System Regulation and Innovative Therapeutics) from the Japanese Ministry of Education, Culture, Sports, Science, and Technology; and Grants from the Hama- guchi Foundation for the Advancement of Biochemistry (to Noritaka Y.) and the Japan Foundation for Applied Enzymology (to Naoto Y.). M.M. and T.H. are Research Assistants of Program for Leading Graduate Schools and Research Fellows of the Japan Society for the Promotion of Science.

Conflict of Interest The authors declare no conflict of interest.

Supplementary Materials The online version of this article contains supplementary materials.

\section{REFERENCES}

1) Tam WL, Weinberg RA. The epigenetics of epithelial-mesenchymal plasticity in cancer. Nat. Med., 19, 1438-1449 (2013). 
2) De Craene B, Berx G. Regulatory networks defining EMT during cancer initiation and progression. Nat. Rev. Cancer, 13, 97-110 (2013).

3) Fischer KR, Durrans A, Lee S, Sheng J, Li F, Wong ST, Choi H, El Rayes T, Ryu S, Troeger J, Schwabe RF, Vahdat LT, Altorki NK, Mittal V, Gao D. Epithelial-to-mesenchymal transition is not required for lung metastasis but contributes to chemoresistance. Nature, 527, 472-476 (2015).

4) Zheng X, Carstens JL, Kim J, Scheible M, Kaye J, Sugimoto H, Wu CC, LeBleu VS, Kalluri R. Epithelial-to-mesenchymal transition is dispensable for metastasis but induces chemoresistance in pancreatic cancer. Nature, 527, 525-530 (2015).

5) Lamouille $\mathrm{S}, \mathrm{Xu}$ J, Derynck R. Molecular mechanisms of epithelial-mesenchymal transition. Nat. Rev. Mol. Cell Biol., 15, 178-196 (2014).

6) Nieto MA. The snail superfamily of zinc-finger transcription factors. Nat. Rev. Mol. Cell Biol., 3, 155-166 (2002).

7) Bernardo GM, Keri RA. FOXA1: a transcription factor with parallel functions in development and cancer. Biosci. Rep., 32, 113-130 (2012).

8) Kaestner KH. The FoxA factors in organogenesis and differentiation. Curr. Opin. Genet. Dev., 20, 527-532 (2010).

9) Carroll JS, Liu XS, Brodsky AS, Li W, Meyer CA, Szary AJ, Eeckhoute J, Shao W, Hestermann EV, Geistlinger TR, Fox EA, Silver PA, Brown M. Chromosome-wide mapping of estrogen receptor binding reveals long-range regulation requiring the forkhead protein FoxA1. Cell, 122, 33-43 (2005).

10) Yamaguchi $N$, Shibazaki M, Yamada C, Anzai E, Morii M, Nakayama Y, Kuga T, Hashimoto Y, Tomonaga T, Yamaguchi N. Tyrosine phosphorylation of the pioneer transcription factor FoxA1 promotes activation of estrogen signaling. J. Cell. Biochem., 118, 1453-1461 (2017).

11) Bernardo GM, Lozada KL, Miedler JD, Harburg G, Hewitt SC, Mosley JD, Godwin AK, Korach KS, Visvader JE, Kaestner KH, Abdul-Karim FW, Montano MM, Keri RA. FOXA1 is an essential determinant of ER $\alpha$ expression and mammary ductal morphogenesis. Development, 137, 2045-2054 (2010).

12) Yamaguchi N, Ito E, Azuma S, Honma R, Yanagisawa Y, Nishikawa A, Kawamura M, Imai J, Tatsuta K, Inoue J, Semba K, Watanabe S. FoxA1 as a lineage-specific oncogene in luminal type breast cancer. Biochem. Biophys. Res. Commun., 365, 711-717 (2008).

13) Song Y, Washington MK, Crawford HC. Loss of FOXA1/2 is essential for the epithelial-to-mesenchymal transition in pancreatic cancer. Cancer Res., 70, 2115-2125 (2010).

14) Jin HJ, Zhao JC, Ogden I, Bergan RC, Yu J. Androgen receptor-independent function of FoxA1 in prostate cancer metastasis. Cancer Res., 73, 3725-3736 (2013).

15) Bernardo GM, Bebek G, Ginther CL, Sizemore ST, Lozada KL, Miedler JD, Anderson LA, Godwin AK, Abdul-Karim FW, Slamon DJ, Keri RA. FOXA1 represses the molecular phenotype of basal breast cancer cells. Oncogene, 32, 554-563 (2013).

16) Thorat MA, Marchio C, Morimiya A, Savage K, Nakshatri H, ReisFilho JS, Badve S. Forkhead box A1 expression in breast cancer is associated with luminal subtype and good prognosis. J. Clin. Pathol., 61, 327-332 (2008).

17) Yamaguchi N, Nakayama $Y$, Yamaguchi N. Down-regulation of Forkhead Box Protein A1 (FOXA1) leads to cancer-stem cell-like properties in tamoxifen-resistant breast cancer cells through induction of interleukin-6. J. Biol. Chem., 292, 8136-8148 (2017).

18) Yamaguchi N, Yuki R, Kubota S, Aoyama K, Kuga T, Hashimoto Y, Tomonaga T, Yamaguchi N. c-Abl-mediated tyrosine phosphorylation of JunB is required for Adriamycin-induced expression of p21. Biochem. J., 471, 67-77 (2015).

19) Aoyama K, Fukumoto Y, Ishibashi K, Kubota S, Morinaga T, Horiike Y, Yuki R, Takahashi A, Nakayama Y, Yamaguchi N. Nuclear c-Abl-mediated tyrosine phosphorylation induces chromatin structural changes through histone modifications that include H4K16 hypoacetylation. Exp. Cell Res., 317, 2874-2903 (2011).

20) Liu YN, Lee WW, Wang CY, Chao TH, Chen Y, Chen JH. Regulatory mechanisms controlling human E-cadherin gene expression. Oncogene, 24, 8277-8290 (2005).

21) Ye Y, Xiao Y, Wang W, Yearsley K, Gao JX, Shetuni B, Barsky SH. $\mathrm{ER} \alpha$ signaling through slug regulates E-cadherin and EMT. Oncogene, 29, 1451-1462 (2010).

22) Shenoy A, Blelloch RH. Regulation of microRNA function in somatic stem cell proliferation and differentiation. Nat. Rev. Mol. Cell Biol., 15, 565-576 (2014)

23) Pan Y, Li J, Zhang Y, Wang N, Liang H, Liu Y, Zhang CY, Zen K, $\mathrm{Gu} \mathrm{H}$. Slug-upregulated miR-221 promotes breast cancer progression through suppressing E-cadherin expression. Sci. Rep., 6, 25798 (2016).

24) Malik S, Jiang S, Garee JP, Verdin E, Lee AV, O'Malley BW, Zhang M, Belaguli NS, Oesterreich S. Histone deacetylase 7 and FoxA1 in estrogen-mediated repression of RPRM. Mol. Cell. Biol., 30, 399-412 (2010). 